\title{
Orbital evolution around irregular bodies
}

\author{
A. Rossi ${ }^{1,2}$, F. Marzari ${ }^{3}$, and P. Farinella ${ }^{4}$ \\ ${ }^{1}$ CNUCE-CNR, Via S. Maria 36, 56126, Pisa, Italy \\ ${ }^{2}$ Université de Paris VII - Obs. de Paris, DESPA, 92195 Meudon Principal cedex, France \\ ${ }^{3}$ Dipartimento di Fisica, Università di Padova, Via Marzolo 8, 35131 Padova, Italy \\ ${ }^{4}$ Dipartimento di Matematica, Università di Pisa, Via Buonarroti 2, 56127 Pisa, Italy
}

(Received October 12, 1998; Revised April 20, 1999; Accepted May 10, 1999)

\begin{abstract}
The new profiles of the space missions aimed at asteroids and comets, moving from fly-bys to rendezvous and orbiting, call for new spaceflight dynamics tools capable of propagating orbits in an accurate way around these small irregular objects. Moreover, interesting celestial mechanics and planetary science problems, requiring the same sophisticated tools, have been raised by the first images of asteroids (Ida/Dactyl, Gaspra and Mathilde) taken by the Galileo and NEAR probes, and by the discovery that several near-Earth asteroids are probably binary. We have now developed two independent codes which can integrate numerically the orbits of test particles around irregularly shaped primary bodies. One is based on a representation of the central body in terms of "mascons" (discrete spherical masses), while the other one models the central body as a polyhedron with a variable number of triangular faces. To check the reliability and performances of these two codes we have performed a series of tests and compared their results. First we have used the two algorithms to calculate the gravitational potential around non-spherical bodies, and have checked that the results are similar to each other and to those of other, more common, approaches; the polyhedron model appears to be somewhat more accurate in representing the potential very close to the body's surface. Then we have run a series of orbit propagation tests, integrating several different trajectories of a test particle around a sample ellipsoid. Again the two codes give results in fair agreement with each other. By comparing these numerical results to those predicted by classical perturbation formulae, we have noted that when the orbit of the test particle gets close to the surface of the primary, the analytical approximations break down and the corresponding predictions do not match the results of the numerical integrations. This is confirmed by the fact that the agreement gets better and better for orbits farther away from the primary. Finally, we have found that in terms of CPU time requirements, the performances of the two codes are quite similar, and that the optimal choice probably depends on the specific problem under study.
\end{abstract}

\section{Introduction}

The first images of asteroidal bodies (Ida, Gaspra and Mathilde) taken by the Galileo and NEAR probes have shown very irregularly shaped objects. A small satellite, Dactyl, was also discovered around Ida (Chapman et al., 1995). These findings, along with ground-based observations indicating the existence of other asteroidal binaries (Pravec and Hahn, 1997; Pravec et al., 1998), have stimulated the study of orbital dynamics around such irregular bodies; in particular the existence of stable orbits allowing for the long-term survival of satellites has been investigated in some detail (Scheeres et al., 1996; Geissler et al., 1997; Petit et al., 1997; Scheeres et al., 1998a).

The first close images of asteroids were taken during fast fly-bys, but the new space missions profiles include now rendezvous with and orbiting around asteroids and comets. The NEAR probe is going to orbit the relatively large near-Earth asteroid Eros for an extended period of time, the Japanese MUSES-C spacecraft is due to rendezvous with the asteroid 4660 Nereus; even more challenging is the concept of the

Copy right (C) The Society of Geomagnetism and Earth, Planetary and Space Sciences (SGEPSS); The Seismological Society of Japan; The Volcanological Society of Japan; The Geodetic Society of Japan; The Japanese Society for Planetary Sciences.
Rosetta mission, where the spacecraft is due to orbit the small nucleus of active comet Wirtanen for more than 8 months. Both these missions require new models and tools to predict and control the navigation and dynamical evolution of an orbiter around a very irregular body in its complex gravity field. Non-gravitational perturbations must also be taken into account, since in particular comets can perturb the spacecraft's orbit with their intense outgassing.

We have now developed two separate software tools to propagate numerically orbits around irregular bodies. One is based on a representation of the central body in terms of "mascons" (discrete spherical masses), while the other models the central body as a homogeneous polyhedron with a variable number of triangular faces. Different gravitational (third-body) and non-gravitational perturbations can also be included. Our final goal is to have a set of dynamical models implemented in a software suite allowing for the thorough study of the dynamics of orbits around asteroids and comets, both from both a theoretical and a more operative (space missions design and navigation) point of view.

In the most recent phase of this project, we have been validating and comparing the two models with relatively easy, yet significant, test cases. In this paper we provide a progress 
report on this work, describing the models and the comparison tests that we have performed so far. In the near future we are going to complete the testing phase and also to include in our software packages new, more refined, models for the non-gravitational perturbations acting around active comet nuclei.

\section{Gravitational Potential Calculations}

The first step of our test campaign has been to assess the accuracy of the gravitational potential representation outside a body of given shape. We have compared the results obtained by calculating the potential outside an homogeneous ellipsoid by using four different approaches:

- Ivory's approach;

- a spherical harmonics approach;

- a "mascons" approach;

- a polyhedral approach.

In the following subsections we will briefly recall the theory underlying each of these methods and then we will describe the results of the comparison tests.

\subsection{Ivory's approach}

It is possible to compute analytically the gravitational attraction of a homogeneous triaxial ellipsoid. The theory dates back to Newton's Principia, but it received a substantial improvement thanks to the work of Laplace and Ivory (Laplace, 1782; Kellog, 1954). The theory allows one to calculate the potential outside a generic ellipsoid; however, the formulae greatly simplify in the case of an axisymmetric ellipsoid, since in this case the elliptic integrals all reduce to elementary functions (arc tangents) (Broucke and Scheeres, 1994). Therefore, for our purposes we have taken an ellipsoid whose semiaxes $a, b$ and $c$ are such that $a=b>c$ and derived the potential at a point with cartesian coordinates $x, y$ and $z$.

Let us introduce the two quantities:

$$
R=\sqrt{c^{2}+h} \quad \text { and } \quad \lambda=\frac{\sqrt{a^{2}-c^{2}}}{R}
$$

where $h$ is the positive root of Ivory's equation:

$$
\psi=\frac{x^{2}+y^{2}}{a^{2}+h}+\frac{z^{2}}{c^{2}+h}-1=0 .
$$

Defining the four functions of $\lambda$ :

$$
\begin{gathered}
H_{0}=\frac{1}{\lambda} \arctan (\lambda), \\
H_{1}=H_{2}=\frac{1}{\lambda^{3}}\left[\arctan (\lambda)-\frac{\lambda}{1+\lambda^{2}}\right], \\
H_{3}=\frac{2}{\lambda^{3}}(\lambda-\arctan (\lambda)),
\end{gathered}
$$

the potential is given by:

$$
V=\frac{3 \mu}{2 R} H_{0}-\frac{3 \mu}{4 R^{3}}\left(x^{2} H_{1}+y^{2} H_{2}+z^{2} H_{3}\right),
$$

where $\mu$ is the mass of the ellipsoid $M$ times the gravitational constant $G$.

\subsection{Spherical harmonics approach}

Since outside of the attracting mass the gravitational potential is a harmonic function, it can be expanded into a series of spherical harmonics (e.g., Heiskanen and Moritz, 1967; German and Friedlander, 1991), that is

$$
\begin{aligned}
V(r, \theta, \lambda)= & \frac{G M}{r}\left[1+\sum_{n=2}^{\infty} \sum_{m=0}^{n}\left(\frac{R_{e}}{r}\right)^{n} P_{n m}(\sin \theta)\right. \\
& \left.\left(C_{n m} \cos m \lambda+S_{n m} \sin m \lambda\right)\right],
\end{aligned}
$$

where $r, \theta$ and $\lambda$ are the radius, latitude and longitude, respectively, in a coordinate system whose origin is at the center of mass of the body, $R_{e}=a$ is the largest equatorial radius of the central body, $P_{n m}$ are the associated Legendre polynomials, and $C_{n m}$ and $S_{n m}$ are the coefficients of the potential determined by the mass distribution within the body.

Given a homogeneous triaxial ellipsoid with axes $a, b$ and $c$, we can calculate the coefficients taking into account that, for such a symmetric body:

$$
C_{n m}=0 \text { for } n \text { or } m \text { odd }
$$

and

$$
S_{n m}=0 \quad \text { for all } n \text { and } m \text {. }
$$

For our purposes we have stopped the expansion of Eq. (1) to the fourth order, and we get the following coefficients:

$$
\begin{gathered}
C_{20}=\frac{2 c^{2}-\left(a^{2}+b^{2}\right)}{10 R_{e}^{2}}, \\
C_{22}=\frac{a^{2}-b^{2}}{20 R_{e}^{2}}, \\
C_{40}=3 \frac{3\left(a^{4}+b^{4}\right)+8 c^{4}+2 a^{2} b^{2}-8\left(a^{2}+b^{2}\right) c^{2}}{140 R_{e}^{4}}, \\
C_{42}=\frac{\left(a^{2}-b^{2}\right)\left(2 c^{2}-a^{2}-b^{2}\right)}{280 R_{e}^{4}}, \\
C_{44}=\frac{\left(a^{2}-b^{2}\right)^{2}}{2240 R_{e}^{4}} .
\end{gathered}
$$

Finally, by expanding to fourth order Eq. (1), we obtain the following expression for the potential:

$$
\begin{aligned}
V=\frac{G M}{r}[1 & +\left(\frac{a}{r}\right)^{2}\left[\frac{1}{2} C_{20}\left(3 \sin ^{2} \theta-1\right)\right. \\
& \left.+3 C_{22} \cos ^{2} \theta \cos (2 \lambda)\right] \\
& +\left(\frac{a}{r}\right)^{4}\left[\frac{1}{8} C_{40}\left(35 \sin ^{4} \theta-30 \sin ^{2} \theta+3\right)\right. \\
& +\frac{15}{2} C_{42} \cos ^{2} \theta\left(7 \sin ^{2} \theta-1\right) \cos (2 \lambda) \\
& \left.\left.+105 C_{44} \cos ^{4} \theta \cos (4 \lambda)\right]\right]
\end{aligned}
$$

Again, we have applied these formulae to the special case of an axisymmetric ellipsoid. 


\subsection{The mascons approach}

This approach, very simple from a conceptual point of view, has been devised in order to calculate the gravitational attraction of bodies with a very irregular shape. Here a body of given shape is approximated by a set of point masses (mascons) placed in a suitable way in order to reproduce the body's mass distribution (Geissler et al., 1997; Scheeres et $a l ., 1998 b)$. In general, the volume circumscribed by the body is filled with a grid of point masses (adding up to the given total mass) and the force exerted on an orbiting particle is computed as the vector sum of the forces due to each point mass.

\subsection{Polyhedral approach}

Another way to deal with very irregularly shaped objects is described in Werner (1994) and Werner and Scheeres (1997). Any body of arbitrary shape can be approximated with a polyhedron having a variable number of faces. Werner developed a theory which allows the calculation, in an analytic form, of the gravitational potential due to a homogeneous polyhedron having triangular faces.

Without going into details (which can be found in Werner and Scheeres (1997)), we give a short description of the method. Given a point at a distance $r$ from the center of a mass $M$, by using Gauss's divergence theorem the potential can be written as:

$$
U=G \iiint_{M} \frac{1}{r} d m=\frac{1}{2} G \rho \iint_{S} \hat{\mathbf{n}} \cdot \hat{\mathbf{r}} d S,
$$

where $\rho$ is the density (assumed to be constant), $S$ is the surface of the body and $\hat{\mathbf{n}}$ is a unit vector normal to the surface element $d S$. If we consider the specific case of a polyhedron, Eq. (2) can be written as a sum over all the faces of the polyhedron:

$$
U=\frac{1}{2} G \rho \sum_{f \in \text { faces }} \hat{\mathbf{n}}_{f} \cdot \mathbf{r}_{f} \iint_{f} \frac{1}{r} d S,
$$

where $\hat{\mathbf{n}}_{f}$ is the unit vector normal to each face and $\mathbf{r}_{f}$ is a vector from the field point to a given point in the face plane.

After some algebra, we obtain the final expression for the potential of the polyhedron:

$$
\begin{aligned}
U= & \frac{1}{2} G \rho \sum_{e \in e d g e s} \mathbf{r}_{e} \cdot \mathbf{E}_{e} \cdot \mathbf{r}_{e} \cdot L_{e} \\
& -\frac{1}{2} G \rho \sum_{f \in \text { faces }} \mathbf{r}_{f} \cdot \mathbf{F}_{f} \cdot \mathbf{r}_{f} \cdot \omega_{f},
\end{aligned}
$$

where the first sum extends over all the edges and the second one over all the faces of the polyhedron. $\mathbf{r}_{e}$ is a vector from the field point to each edge, $\mathbf{E}_{e}$ is a dyad defined in terms of the face and edge normal vectors associated with each edge, $L_{e}$ is a logarithmic term expressing the potential of a 1-D straight wire, $\mathbf{F}_{f}$ is a dyad defined for each face as the outer product of the face unit normal vector with itself and, finally, $\omega_{f}$ is the signed solid angle subtended by a face when viewed from the field point.

We have implemented this algorithm in a code which first allows for the construction of sample polyhedra by successive deformations of a sphere, approximated as a polyhedron, with the number of triangular faces variable according to the desired accuracy of the body's representation. Then it is possible to integrate orbits around this body by taking into account, besides its gravitational attraction, third-body perturbations and non-gravitational forces (so far, we have included the solar radiation pressure and the Poynting-Robertson effect) (Rossi and Fulchignoni, 1998).

\section{Results of the Comparison Tests}

In order to assess the accuracy of the gravitational potential representations obtained with the methods described above, we have calculated the potential outside two homogeneous ellipsoids: the first is an axisymmetric ellipsoid with $a=$ $b=10 \mathrm{~km}$ and $c=5 \mathrm{~km}$ and the second one is a triaxial ellipsoid with $a=30 \mathrm{~km}, b=10 \mathrm{~km}$ and $c=6.666 \mathrm{~km}$. We assumed a comet-like density $\rho=1 \mathrm{~g} / \mathrm{cm}^{3}$, so that the mass of the two bodies are $M_{1}=2.0943951 \times 10^{15} \mathrm{~kg}$ and $M_{2}=8.3775803 \times 10^{15} \mathrm{~kg}$.

Then we have generated the same ellipsoids with the mascons and the polyhedral approaches; due to the approximations involved in the generating procedure the actual masses of the bodies used in the comparison have been: $M_{1}=$ $2.083095 \times 10^{15} \mathrm{~kg}$ and $M_{2}=8.29181 \times 10^{15} \mathrm{~kg}$. In line of principle, of course, it is possible to match the masses resulting from the numerical approaches to the "theoretical" values reported above - this was not done in this preliminary phase of our work, but we thought that, for the time being, comparisons at the $1 \%$ level are enough for our purposes. In the polyhedron approach both the objects had 1521 faces while in the mascons one we used about 6000 point masses.

\subsection{Axisymmetric ellipsoid}

The axisymmetric ellipsoid allows us to apply the Ivory approach of Subsection 2.1, which we use as the reference value for the comparison. The potential is calculated along the $x$ and $z$ axes at $40 \mathrm{~m}$ steps, from the surface $(10 \mathrm{~km})$ up to a distance of $30 \mathrm{~km}$.

Figure 1 shows the behavior of the potential along the $z$ axis as a function of the distance. In Fig. 2 we plot directly the difference of the potential values obtained from the dif-

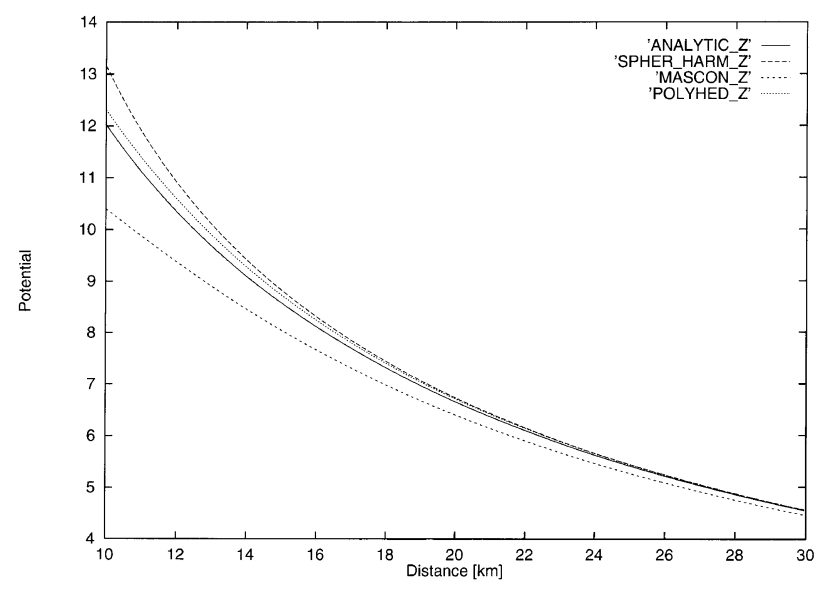

Fig. 1. Potential along the $z$ axis for the axisymmetric ellipsoid calculated with Ivory's approach (solid line), the spherical harmonics approach (dashed line), the mascons approach (small-dashed) and the polyhedral approach (dotted line). 


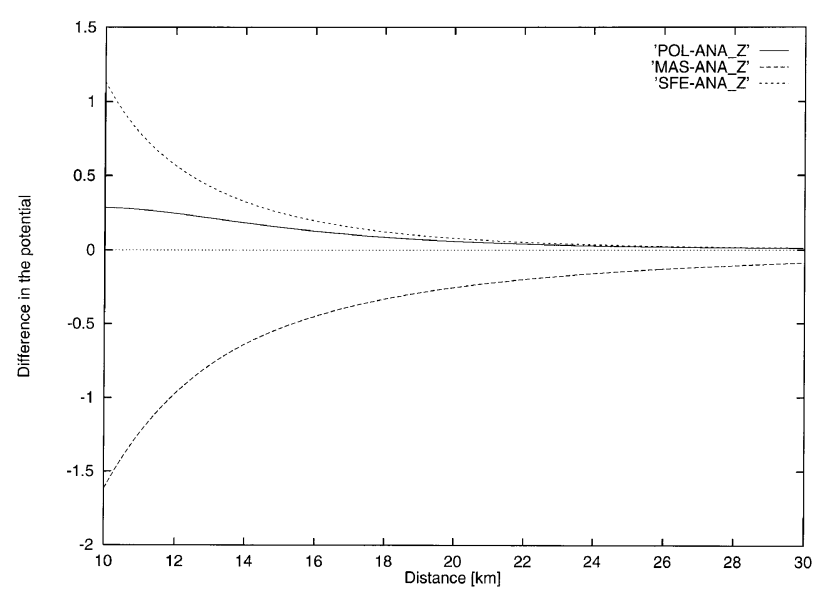

Fig. 2. Difference, with respect to Ivory's approach, of the values of the potential calculated along the $z$ axis from the polyhedral approach (solid line), the mascons approach (dashed line) and the spherical harmonics approach (small-dashed line).

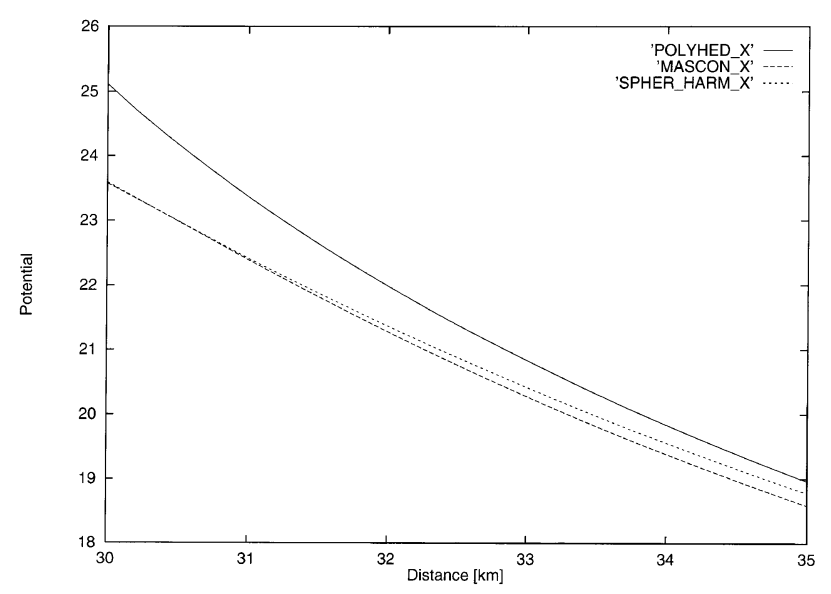

Fig. 3. Potential along the $x$ axis for the triaxial ellipsoid calculated with the polyhedral approach (solid line), the mascons approach (dashed line) and the spherical harmonics approach (small-dashed line).

ferent approaches along the $z$ axis, with respect to the value calculated with the Ivory's approach. All units are taken in the MKS system.

As a first general remark we can say that the four methods give results fairly close to each other. Of course, going far enough from the central body, the effects due to its nonspherical shape decrease and all the results converge to a common line. On the other hand, when we are very close to the surface the polyhedral approach appears to give more accurate results. For the spherical harmonics approach it has to be noted that the series expansion had been truncated quite soon (stopping to the fourth order), while in the mascons case the roughness of the body surface, due to the regular distribution of the discrete point masses, leads to some discrepancy.

\subsection{Triaxial ellipsoid}

A more general test can be performed in the case of a triaxial ellipsoid. However, since in this case we cannot apply the analytical Ivory approach described in Subsection 2.1, our comparison is limited to the other three approaches, without

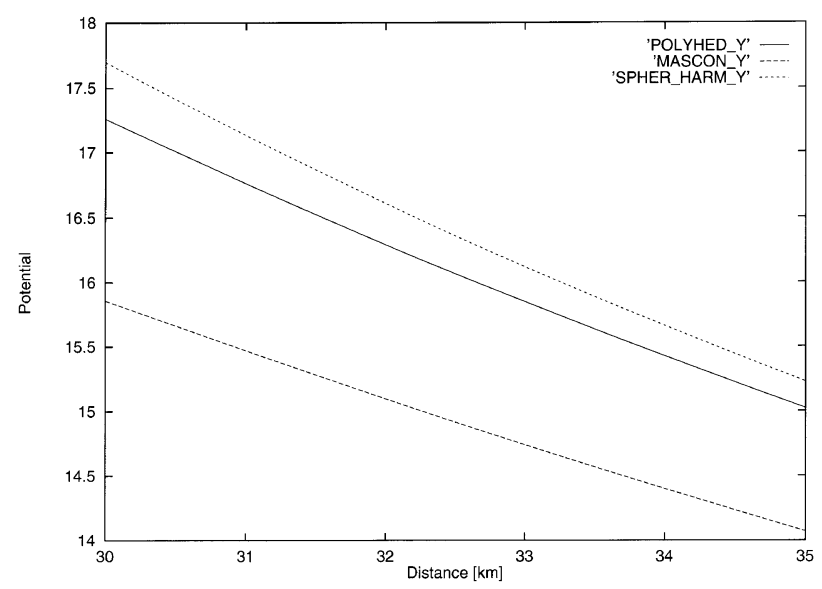

Fig. 4. Potential along the $y$ axis for the triaxial ellipsoid calculated with the polyhedral approach (solid line), the mascons approach (dashed line) and the spherical harmonics approach (small-dashed line).

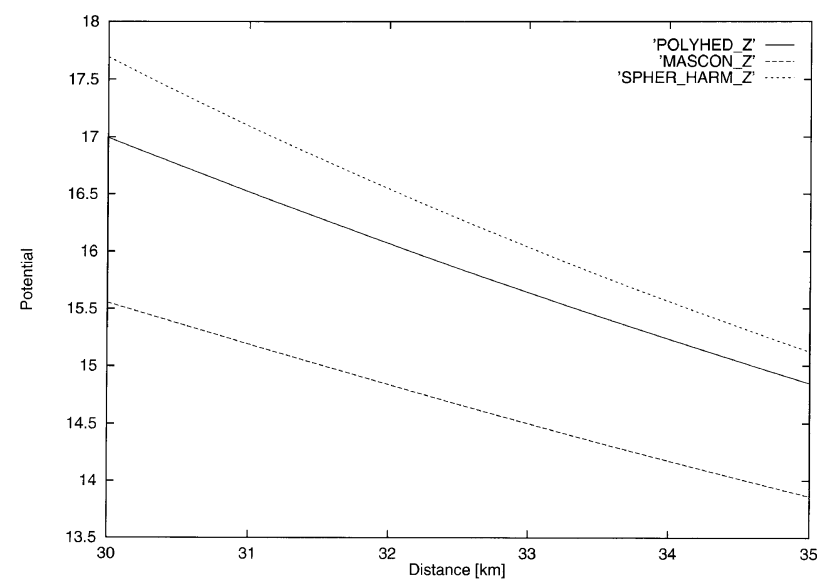

Fig. 5. Potential along the $z$ axis for the triaxial ellipsoid calculated with the polyhedral approach (solid line), the mascons approach (dashed line) and the spherical harmonics approach (small-dashed line).

adopting a reference one. The potential is calculated along the $x, y$ and $z$ axes at $100 \mathrm{~m}$ steps, starting on or near the surface (at $r=30 \mathrm{~km}$ ) up to a distance of $80 \mathrm{~km}$.

Figures 3-5 show the value of the potential in the region close to the surface calculated with the three different approaches, along the three cartesian axes centered on the body's center of mass. At large distances, again, the three values quickly converge toward a common line. We can note that the different methods give very similar results; those of the polyhedral and spherical harmonics approaches are generally fairly close to each other, while on most cases the mascons approach shows a more marked (but still small) discrepancy in the region close to the surface.

\section{Orbit Propagations}

We have used the mascons and polyhedral approaches to build two independent codes capable of propagating orbits around irregular bodies. First they proceed to the construction of sample objects either by filling a grid with point masses or by successive deformations of a sphere, approx- 
Table 1. Semimajor axis $(a$, in km)), eccentricity $(e)$, inclination $(i)$, argument of the ascending node $(\Omega)$, argument of pericenter $(\omega)$ and mean anomaly $(M)$ for the test propagations.

\begin{tabular}{lcccccc}
\hline \multicolumn{7}{c}{ Initial orbital elements for the test propagations } \\
\hline Test & $a$ & $e$ & $i$ & $\Omega$ & $\omega$ & $M$ \\
\hline Sphere & $19.989 \mathrm{~km}$ & 0.000356 & $10^{\circ}$ & $0.0389^{\circ}$ & $174.6594^{\circ}$ & $18.3446^{\circ}$ \\
Ellipsoid (inclined) & 19.987 & 0.000645 & $10^{\circ}$ & $0^{\circ}$ & $180^{\circ}$ & $180^{\circ}$ \\
Ellipsoid (incl./ell.) & 19.981 & 0.199226 & $10^{\circ}$ & $0^{\circ}$ & $0^{\circ}$ & $0^{\circ}$ \\
Ellipsoid (distant) & 39.961 & 0.199226 & $10^{\circ}$ & $0^{\circ}$ & $0^{\circ}$ & $0^{\circ}$ \\
\hline
\end{tabular}

imated as a polyhedron, with a number of triangular faces variable according to the desired accuracy of the body's representation. Then, the orbits of test particles are propagated using, in both the codes, the RA15 version of the numerical integrator RADAU (Everhart, 1985) to integrate numerically the equations of motion. Besides the gravitational attraction of the irregular body (calculated with either the mascons or the polyhedral approach), third-body perturbations and some non-gravitational forces (currently, solar radiation pressure and the Poynting-Robertson effect) can be computed (Rossi and Fulchignoni, 1998). The rotation of the primary around a principal axis of inertia can also be accounted for.

After having verified the reliability of the potential calculations with the two different approaches, as described in Section 3, we have performed a systematic comparison of the results of several orbit propagation test cases. Since in this phase of our work we were mainly interested in a validation of the two codes, we have selected a few simple test cases, considering only the gravitational attraction of the central body and no additional perturbation. We also compared the results of the two codes with those obtained by means of a "standard" orbital propagator using the spherical harmonics approach. We have always adopted a propagation time span of 60 days. In Table 1 we have listed the initial orbital elements of the four test propagations described in the remainder of this section.

\subsection{Sphere with inclined, circular orbit}

The first test case was the propagation of a circular inclined $\operatorname{orbit}\left(a=19.989 \mathrm{~km}, e=0.000356, i=10^{\circ}, \Omega=0.0389^{\circ}\right.$, $\left.\omega=174.6594^{\circ}, M=18.3446^{\circ}\right)$ around a spherical primary. We have considered a sphere with a radius $R=10 \mathrm{~km}$ and a density $\rho=1 \mathrm{~g} / \mathrm{cm}^{3}$. This sphere was approximated using either 11753 mascons or a polyhedron with 1521 faces. The mass of this "quasi-sphere" was $4.196 \times 10^{15} \mathrm{~kg}$.

Figure 6 shows a 3-D plot of the initially circular and inclined orbit around the sample polyhedron, and Fig. 7 shows the corresponding semimajor axis evolution. Both in this case and for the same orbit integrated with the mascons model, the semimajor axis and eccentricity show shortperiodic variations of the order of a few tens of $\mathrm{cm}$ in $a$ and a few parts in $10^{-4}$ in $e$. Filtering out these short-term perturbations, the two codes perform properly in this case, yielding the same five constant orbital elements as output as they had been given in input, at least down to a relative error less than $10^{-6}$ (see Fig. 7).

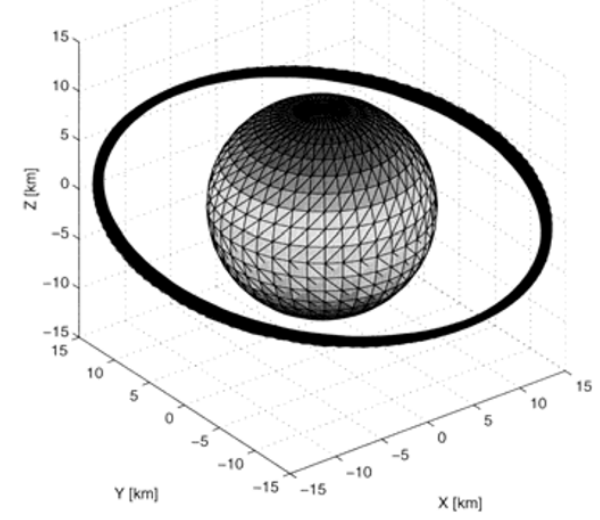

Fig. 6. Circular inclined orbit around the spherical primary, modelled with the polyhedral approach.

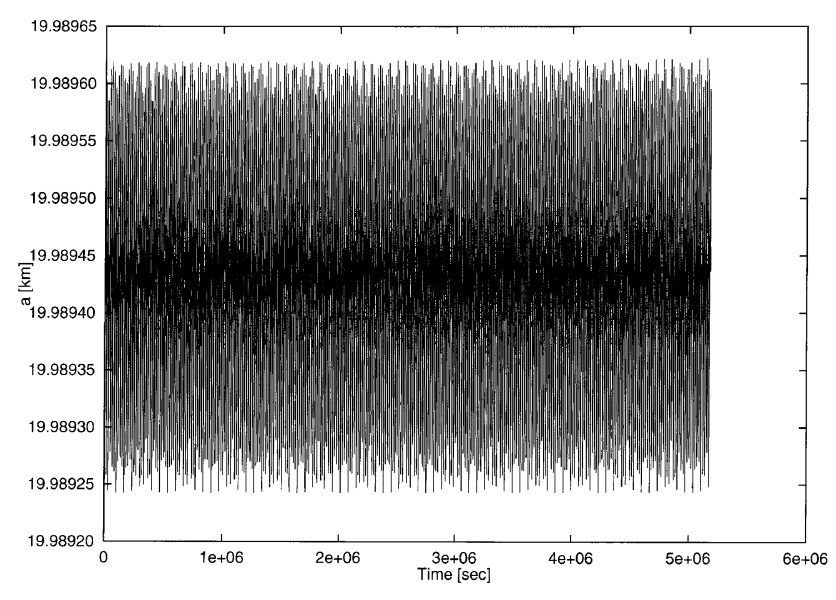

Fig. 7. Semimajor axis vs. time for the test case with a circular orbit around a sphere.

\subsection{Ellipsoid with inclined, circular orbit}

In this test the central body was an axisymmetric ellipsoid with semiaxes: $a=b=10 \mathrm{~km}$ and $c=5 \mathrm{~km}$. It has been approximated either by 5835 mascons or by a polyhedron with 1521 faces. The total mass is $2.083 \times 10^{15} \mathrm{~kg}$, corresponding again to a density $\rho=1 \mathrm{~g} / \mathrm{cm}^{3}$.

First we have compared the periods of the numerically integrated orbit (with initial elements: $a=19.987 \mathrm{~km}, e=$ 
$0.000645, i=10^{\circ}, \Omega=0^{\circ}, \omega=180^{\circ}, M=180^{\circ}$ ), obtained from both the codes, with the theoretical one derived from perturbation theory. For a satellite in a circular orbit about an oblate planet one can write a modified version of Kepler's third law (Roy, 1982), as follows:

$$
T=2 \pi\left\{\frac{r^{3}}{G M}\left[1-\left(\frac{R}{r}\right)^{2} \epsilon\right]\right\}^{1 / 2},
$$

where $R$ is the body's equatorial radius and

$$
\epsilon=3 \frac{(C-A)}{2 M R^{2}},
$$

with $C$ and $A$ the moments of inertia of the body with respect to the $z$ and $x$ axes. In our test case Eq. (4) yields $T=46305 \mathrm{~s}$. On the other hand, the periods of the integrated orbits are $T_{p} \simeq 45256 \mathrm{~s}$ and $T_{m} \simeq 45220$ (hereinafter the subscripts $p$ and $m$ will denote results from the polyhedral and the mascons approach, respectively). Thus the periods of the integrated orbits are very close to each other (the discrepancy is smaller than $0.1 \%$ ), whereas the difference from the theoretical value is about $2 \%$. This is already a clear indication that for orbits so close to a strongly non-spherical primary the assumptions implicit in the perturbation equations are not fulfilled to a very high accuracy.

For the nodal rate the classical result from first-order secular perturbation theory is:

$$
\frac{d \Omega}{d t}=-\frac{3}{2} \frac{J_{2} R^{2}}{p^{2}} \bar{n} \cos i
$$

where $p=a\left(1-e^{2}\right)$ and

$$
\bar{n}=n_{0}\left[1+\frac{3}{2} \frac{J_{2} R^{2}}{p^{2}}\left(1-\frac{3}{2} \sin ^{2} i\right)\left(1-e^{2}\right)^{1 / 2}\right]
$$

is the perturbed mean motion of a particle moving around an oblate body and $n_{0}$ is the unperturbed value. The $J_{2}$ coefficient is given by:

$$
J_{2}=\frac{C-\frac{A+B}{2}}{M R^{2}}=\frac{\left(a^{2}-c^{2}\right)}{5 R^{2}}=0.15
$$

where $B$ is the moment of inertia with respect to the $y$ axis. We have used the fact that for an axisymmetric ellipsoid $A=B=M\left(a^{2}+c^{2}\right) / 5$. This value of $J_{2}$ can be compared to the value easily computed numerically for the mascons model, for which we have obtained: $A=B=5.206 \times 10^{22}$ $\mathrm{kg} \mathrm{m}^{2}$ and $C=8.348 \times 10^{22} \mathrm{~kg} \mathrm{~m}^{2}$ and $J_{2}=0.15084$. Thus the error implicit in the mascons representation is of the order of $0.6 \%$. The analytical value of $J_{4}$ is 0.0964 .

From Eq. (5) the theoretical nodal rate is $d \Omega / d t=-7.7 \times$ $10^{-6} \mathrm{rad} \mathrm{s}^{-1}$. On the other hand for the integrated orbits we have obtained: $d \Omega_{p} / d t \simeq-1.09 \times 10^{-5} \mathrm{rad} \mathrm{s}^{-1}$ and $d \Omega_{m} / d t \simeq-1.11 \times 10^{-5} \mathrm{rad} \mathrm{s}^{-1}$, again close to each other (within 2\%) but with a much greater difference (about 30\%) with respect to the theoretical value given above.

We calculated $\dot{\Omega}$ from the orbits integrated with the spherical harmonic approach; first using only $J_{2}$ and then $J_{2}$ and $J_{4}$. The values obtained are $d \Omega_{J 2} / d t \simeq-7.3 \times 10^{-6} \mathrm{rad}$ $\mathrm{s}^{-1}$ and $d \Omega_{J 4} / d t \simeq-1.01 \times 10-5 \mathrm{rad} \mathrm{s}^{-1}$, respectively.
The value $d \Omega_{J 2} / d t$ is obviously very close to the theoretical one since the latter is calculated taking into account precisely only this term. On the other hand, $d \Omega_{J 4} / d t$ approaches the values obtained from the polyhedral and the mascons models, $d \Omega_{p} / d t$ and $d \Omega_{m} / d t$. The difference between the values obtained using only the $J_{2}$ term and that with $J_{2}$ and $J_{4}$ shows that the spherical harmonics expansion is slowly converging for such highly elongated bodies; therefore it is necessary to calculate a large number of coefficients to have a realistic picture of the gravitational field. This is of course going to be very difficult if one is dealing with a real highly irregular asteroid or comet nucleus.

Figure 8 shows a comparison between the nodal rates derived from the mascons and polyhedral codes.

\subsection{Ellipsoid with inclined, elliptical orbit}

In the previous case the drift rate of the pericenter could not be determined since the orbit was nearly circular. Therefore in the next test we have propagated, around the same ellipsoid, an inclined elliptical orbit ( $a=19.981 \mathrm{~km}, e=$ $0.199226, i=10^{\circ}, \Omega=0^{\circ}, \omega=0^{\circ}, M=0^{\circ}$ ).

From Eq. (5) the theoretical nodal drift rate in this case is $d \Omega / d t=-8.37 \times 10^{-6} \mathrm{rad} \mathrm{s}^{-1}$, whereas for the numerically integrated orbits we have obtained $d \Omega_{p} / d t \simeq-1.25 \times 10^{-5}$ $\operatorname{rad~s}{ }^{-1}, d \Omega_{m} / d t \simeq-1.33 \times 10^{-5} \mathrm{rad} \mathrm{s}^{-1}\left(d \Omega_{J_{4}} / d t \simeq\right.$ $\left.1.15 \times 10^{-5} \mathrm{rad} \mathrm{s}^{-1}\right)$, that is results quite similar to those derived earlier.

As for the pericenter drift rate, the classical formula from perturbation theory is:

$$
\frac{d \omega}{d t}=\frac{3}{4} \frac{J_{2} R^{2}}{p^{2}} \bar{n}\left(1-5 \cos ^{2} i\right) .
$$

which in our specific case yields $d \omega / d t=1.74 \times 10^{-5}$ $\operatorname{rad~} \mathrm{s}^{-1}$. On the other hand, the pericenter rates of the integrated orbits are $d \omega_{p} / d t \simeq 2.90 \times 10^{-5} \mathrm{rad} \mathrm{s}^{-1}$ and $d \omega_{m} / d t \simeq 2.72 \times 10^{-5} \mathrm{rad} \mathrm{s}^{-1}\left(d \omega_{J 4} / d t \simeq 2.21 \times 10^{-5}\right.$ $\operatorname{rad~s} s^{-1}$ ).

Again, the discrepancy between the numerical values (about 6\%) is much smaller than with respect to the value from the analytical formula $(\approx 40 \%)$.

Figure 9 shows a comparison between the pericenter drift

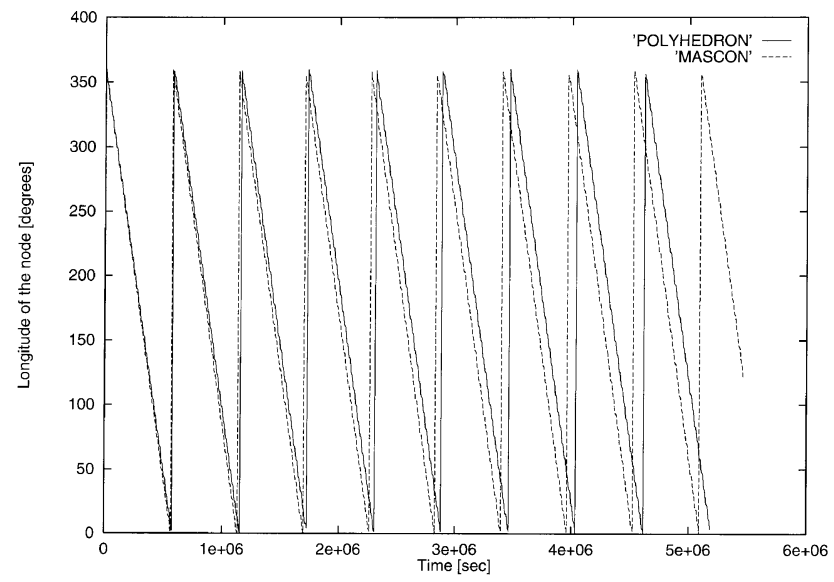

Fig. 8. Nodal drift of the numerically integrated circular orbit around the axisymmetric ellipsoid from the polyhedral (solid line) and the mascons (dashed line) models. 


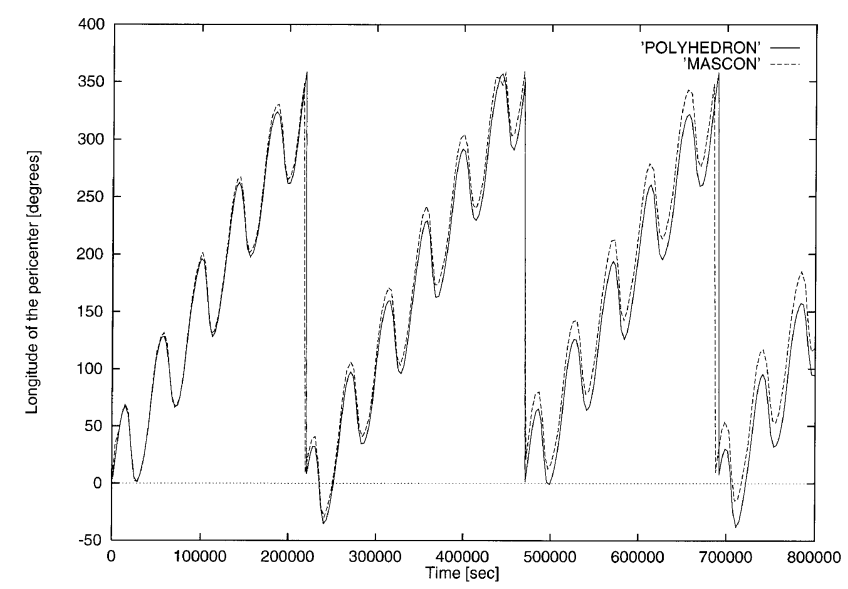

Fig. 9. Pericenter drift of the numerically integrated elliptic orbit around the ellipsoid from the polyhedral (solid line) and the mascons model (dashed line).

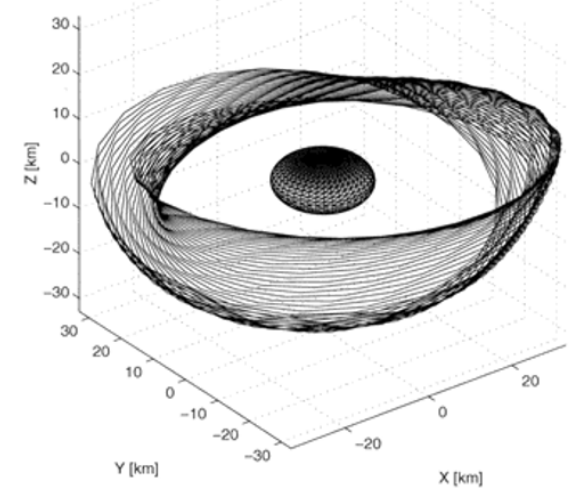

Fig. 10. A 3-D view of the elliptical, inclined, distant orbit around the ellipsoid, approximated with the polyhedral approach.

rates from the polyhedral and mascons codes; short-period effects (whose periodicity coincides with the orbital period) are superimposed to the secular drift.

\subsection{Ellipsoid with inclined, elliptical, distant orbit}

In general the tests described so far show a relatively good agreement between the results from the two numerical models, but large discrepancies with respect to the theoretical values. As we have mentioned previously, we have found hints that these discrepancies are due to the fact that the orbits integrated in the previous cases stay very close to the surface of the ellipsoid; in this case the effects due to the strongly non-spherical shape of the primary cannot be treated as a small perturbation of the two-body problem. In order to test this tentative conclusion, we have considered a test particle moving farther away from the central body, on an inclined elliptical orbit. The orbital elements are: $a=39.961 \mathrm{~km}$, $e=0.199226, i=10^{\circ}, \Omega=0^{\circ}, \omega=0^{\circ}, M=0^{\circ}$. Figure 10 shows a 3-D plot of this orbit around the sample polyhedron.

In this case the theoretical nodal rate is $d \Omega / d t=-7.10 \times$ $10^{-7} \mathrm{rad} \mathrm{s}^{-1}$, while those of the integrated orbits are: $d \Omega_{p} / d t \simeq-7.76 \times 10^{-7} \mathrm{rad} \mathrm{s}^{-1}$ and $d \Omega_{m} / d t \simeq-7.92 \times$

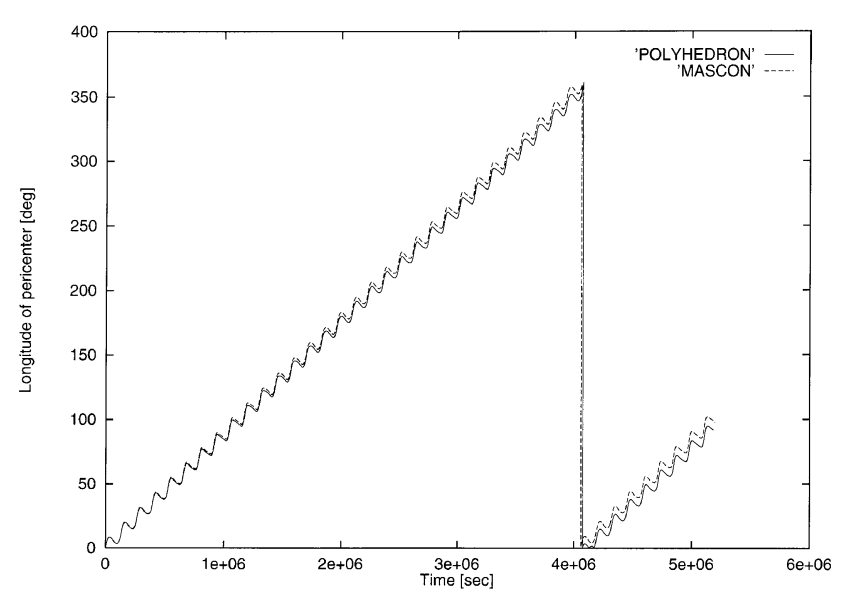

Fig. 11. Pericenter rate of the distant orbit around the ellipsoid from the polyhedral (solid line) and the mascons model (dashed line).

$10^{-7} \mathrm{rad} \mathrm{s}^{-1}$, with discrepancies of $8 \%$ and $10 \%$ respectively $\left(d \Omega_{J 4} / d t \simeq-7.79 \times 10^{-7} \mathrm{rad} \mathrm{s}^{-1}\right)$. The theoretical pericenter drift rate is $d \omega / d t=1.42 \times 10^{-6} \mathrm{rad} \mathrm{s}^{-1}$, to be compared to $d \omega_{p} / d t \simeq 1.53 \times 10^{-6} \mathrm{rad} \mathrm{s}^{-1}$ and $d \omega_{m} / d t \simeq 1.55 \times 10^{-6}$ $\mathrm{rad} \mathrm{s}^{-1}$, that is discrepancies of $8 \%$ and $10 \%$ respectively $\left(d \omega_{J 4} / d t \simeq 1.51 \times 10^{-6} \mathrm{rad} \mathrm{s}^{-1}\right)$. The pericenter drifts derived from the polyhedral and mascons approaches are shown in Fig. 11.

The improved agreement between the theoretical values and the results of the numerical integration confirms that our starting hypothesis is probably correct: orbiting farther away from the central body the perturbations due to the body's shape are reduced and the analytical formulae provide better approximations.

\section{Conclusions and Future Work}

We have described the first phase of our work, which was mainly devoted to the validation of the two software packages. The tests and comparison discussed above in our opinion show their reliability. They can accurately represent the gravitational potential around non-spherical bodies, and this allows one to integrate numerically the orbits of test particles around these objects. The discrepancies between the results of the two codes, as far as the most important secular perturbations are concerned, are typically of the order of a few percent. The situation is most critical for orbits staying close to the surface of the primary, which probably require a higher resolution of the models (in terms of the adopted number of mascons or faces) to achieve an acceptable accuracy.

Another interesting result from our work has been that the classical formulae from the perturbation theory have a very poor accuracy whenever the shape of the primary is strongly non-spherical and the orbital distance is as small as several radii of the primary. In these cases numerical integrations appear really needed for a quantitative study of the secular effects.

Finally, we have checked the CPU time requirements of the two different numerical algorithms. Of course these depend on the number of faces or mascons used to represent the central body (see e.g., figure 3 of Rossi and Fulchignoni (1998)). Nonetheless, some further quantitative conclusions 
can be drawn from the tests described in this paper. With the number of faces/mascons adopted here to describe the ellipsoid in the orbit propagation tests (i.e., 1521 faces and 5835 mascons), the CPU time needed for a 60 days integration of the elliptic orbit on a Pentium II $400 \mathrm{MHz}$ computer was about $1280 \mathrm{~s}$ with the polyhedral model and $1065 \mathrm{~s}$ with the mascons approach. Therefore, with the values adopted for these tests, the mascons code was somewhat faster. On the other hand, we have found hints of a better accuracy of the polyhedral code, especially for orbits getting close to the primary's surface. Of course, in every specific case an optimal trade-off must be found between accuracy and computing speed. It is likely that the trade-off will be different for the two numerical approaches, and that this can lead to select one of the codes for any specific study to be carried out.

As pointed out by Werner and Scheeres (1997), the polyhedral algorithm is suitable for parallelization in an almost natural way; in the future we plan to code it also in Professional Fortran, which allows an easy parallelization of Fortran programs, and this should greatly improve the performances of the code. The same could be done with the mascons code too.

In this phase of our work, we have devoted a particular effort to testing the sections of our software packages related to the calculation of the gravitational potential, since these are the most delicate and the less standard models. However, as we noted before, the codes also include different models for gravitational and non-gravitational perturbations, which have been tested separately and will be used again in the near future.

We already used the codes for a few theoretical studies (Rossi and Fulchignoni, 1998; Scheeres et al., 1998b). Our final aim is to assemble a software suite capable of dealing both with theoretical and with operational issues (such as mission design and navigation), related to the propagation of orbits around small irregular bodies such as asteroids and comets. For the latter class of bodies, we will need to develop suitable models for the outgassing of the comet nucleus and the related drag forces, perturbing the orbital motion of an orbiting probe. Overall, this is an exciting new area of celestial mechanics and astrodynamics, for which times have just become ripe.
Acknowledgments. The authors wish to thank Dr. M. Yoshikawa and Dr. D. Durda for their useful reviews and comments.

\section{References}

Broucke, R. A. and D. J. Scheeres, Computing orbits around an ellipsoid of revolution, paper AAS 94-161, in Advances in the Astronautical Sciences, Spaceflight Mechanics 1994, Vol. 87, Part I, pp. 423-440, 1994.

Chapman, C. R., J. Veverka, P. C. Thomas, K. Klaasen, M. J. S. Belton, A. Harch, A. McEwen, T. V. Johnson, P. Helfenstein, M. E. Davies, W. J. Merline, and T. Denk, Discovery and physical properties of Dactyl, a satellite of the asteroid 243 Ida, Nature, 374, 783-785, 1995.

Everhart, E., An efficient integrator that uses Gauss-Radau spacings, in Dynamics of Comets: Their Origin and Evolution, edited by A. Carusi and G. B. Valsecchi, pp. 185-202, Reidel, 1985.

Kellog, O. D., Foundations of Potential Theory, Dover, 1954.

Geissler, P., J.-M. Petit, D. Durda, R. Greenberg, W. Bottke, M. Nolan, and J. Moore, Erosion and ejecta reaccretion on 243 Ida and its moon, Icarus, 120, 140, 1997.

German, D. and A. L. Friedlander, A simulation of orbits around asteroids using potential field modelling, paper AAS 91-182, in Advances in the Astronautical Sciences, Spaceflight Mechanics 1991, Vol. 75, Part II, pp. 1183-1201, 1991.

Heiskanen, W. A. and H. Moritz, Physical Geodesy, Freeman and Co., 1967. Laplace, P. S., Oeuvres de Laplace ${ }^{\dagger}$, Vol. 2, Chapter 1, 1782.

Petit, J.-M., D. D. Durda, R. Greenberg, T. A. Hurford, and P. E. Geissler, The long-term dynamics of Dactyl's orbit, Icarus, 130, 177-197, 1997.

Pravec, P. and G. Hahn, Two-period lightcurve of $1994 \mathrm{AW}_{1}$ : Indication of a binary asteroid?, Icarus, 127, 431-440, 1997.

Pravec, P., M. Wolf, and L. Šrounovà, Occultation/eclipse events in binary asteroid $1991 \mathrm{VH}$, Icarus, 133, 79-88, 1998.

Rossi, A. and M. Fulchignoni, Study of the environment around the Rosetta candidate target asteroids, Planet. Space Sci., 47(6-7), 873-881, 1999.

Roy, A. E., Orbital Motion, Hilger, Bristol, 1982.

Scheeres, D. J., S. J. Ostro, R. S. Hudson, and R. A. Werner, Orbits close to asteroid 4769 Castalia, Icarus, 121, 67-87, 1996.

Scheeres, D. J., S. J. Ostro, R. S. Hudson, E. M. DeJong, and S. Suzuki, Dynamics of orbits close to asteroid 4179 Toutatis, Icarus, 132, 53-79, 1998 a.

Scheeres, D. J., F. Marzari, L. Tomasella, and V. Vanzani, ROSETTA mission: Satellite orbits around a cometary nucleus, Planet. Space Sci., 46, 649-671, 1998b.

Werner, R. A., The gravitational potential of a homogeneous polyhedron, Celest. Mech. Dyn. Astr., 59, 253-278, 1994.

Werner, R. A. and D. J. Scheeres, Exterior gravitation of a polyhedron derived and compared with harmonic and mascon gravitation representations of asteroid 4769 Castalia, Celest. Mech. Dyn. Astr., 65, 313-344, 1997.

${ }^{\dagger}$ English translation by Bowditch

A. Rossi (e-mail: alessandro.rossi@cnuce.cnr.it), F. Marzari, and P. Farinella 